\title{
Tracers and corresponding detection devices: technetium colloids, blue dyes \& NIR fluorescence
}

\author{
Agnieszka Rychlik ${ }^{1}$, Kamil Zalewski ${ }^{2}$ \\ ${ }^{1}$ Maria Skłodowska-Curie National Research Institute of Oncology in Warsaw, Warsaw, Poland; ${ }^{2}$ Holy Cross Cancer Centre in Kielce, Kielce, Poland \\ Contributions: (I) Conception and design: All authors; (II) Administrative support: None; (III) Provision of study materials or patients: None; (IV) \\ Collection and assembly of data: None; (V) Data analysis and interpretation: None; (VI) Manuscript writing: All authors; (VII) Final approval of \\ manuscript: All authors. \\ Correspondence to: Agnieszka Rychlik. Maria Skłodowska-Curie National Research Institute of Oncology in Warsaw, Warsaw, Poland. \\ Email: agarychlik@gmail.com.
}

\begin{abstract}
Tracers and corresponding detection devices for the mapping of sentinel lymph nodes have been evolving since the first use of lymphangiogram methods in 1977 in penile carcinoma. Nowadays a variety of dyes and radiotracers have been validated for use in breast, vulvar and cervical cancer as well as melanoma. Each tumor site with its anatomical conditions requires different mapping protocol. While the combination of radiotracer and blue dye or radiotracer alone is an established method for breast surgery, vulvar cancer and melanoma, in pelvic sentinel lymph node mapping indocyanine green is currently gaining popularity. Near infrared fluorescence imaging is an emerging technique that enables a real-time image-guided procedure and is currently approved by the Food and Drug Administration as a sentinel lymph node mapping substance with standard of care. New tracers and devices are constantly under investigation to better understand the pathway of lymphatic drainage and increase the sensibility and sensitivity of the method. In the present review the evolution of available tracers and detection devices is discussed. An exhaustive review of current clinical indications of each method, its particularities and adverse effects is made. Finally, an update on ongoing clinical studies in sentinel lymph node mapping methods is presented.
\end{abstract}

Keywords: Sentinel lymph node; technetium colloids; blue dyes; NIR fluorescence

Submitted Dec 16, 2020. Accepted for publication Apr 05, 2021.

doi: $10.21037 / \mathrm{cco}-20-252$

View this article at: http://dx.doi.org/10.21037/cco-20-252

\section{Introduction}

A selection of a correct tracer and detection method is crucial for effective sentinel lymph node mapping. The story begins in 1977 when Cabanas used lymphangiograms method with a radiocontrast agent to successfully map Sentinel Lymph Node (SLN) in penile carcinoma in 100 patients (1). One year later a group from UCLA School of Medicine studied the use of colloidal gold radionucleotide 198Au to show the direction of regional lymphatic drainage in patients with primary malignant melanoma (2). In 1992 Morton and collaborators demonstrated the accuracy of intraoperative lymphatic mapping and selective sentinel lymphadenectomy in patients with primary cutaneous malignant melanoma, using isosulfan and patent blue-v (3). Krag and collaborators first investigated the use of radioisotopes for SLN identification in breast cancer (4). Later, Albertini identified the SLN using a combined technique of blue dye and radioisotope (5).

While the combination of radiotracer and blue dye or radiotracer is an established method for breast surgery, vulvar cancer and melanoma, in pelvic lymph node mapping indocyanine green is currently gaining popularity

New tracers and devices are constantly under investigation to better understand the biology of lymphatic drainage and increase the sensibility and sensitivity of the method. The evolution of available tracers and detection methods and new lines of investigation in sentinel lymph 
node mapping will be discussed in this review.

\section{Vital dyes in SLN mapping}

The colometric technique is simple, relatively cheap and does not require additional detection equipment.

Currently used vital dyes have first been tested on animal models. In pilot studies Cyalume ${ }^{\circledR}$ (visible light fluorescent dye), methylene blue and isosulfan blue were used (6). Cyalume ${ }^{\circledR}$ has not been found useful as it stained surrounding tissues together with lymphatics. Methylene blue was not satisfactory in animal models due to insufficient lymphatic intake. In contrast, isosulfan has been found to be the most promising dye with selective avidity for lymphatic circulation (7).

At present several blue dyes are used in clinical practice. Isosulfan blue is an isomer of the sodium salt-based patent blue $\mathrm{V}$, and is also called lymphazurin. After injection, this dye binds to albumins and is absorbed by the lymphatics, which allows delineation of the lymphatic drainage.

Isosulfan blue for identification of lymphatic vessels has first experimentally been studied in 1980 in rat models with excellent results. The investigators found a sensibility of 97.4\% for identification of lymphatic vessels (7).

This dye has been used in pilot studies by Morton and collaborators to map sentinel lymph nodes in more than five hundred patients with malignant melanoma. The authors demonstrated the accuracy of intraoperative lymphatic mapping and selective sentinel lymphadenectomy to identify lymph node metastasis and reported a less than $1 \%$ false-negative rate (3).

A few years later Giuliano used isosulfan blue to successfully map sentinel lymph nodes in breast carcinoma with a $2.8 \%$ false negative rate. In his study sentinel nodes were identified in 114 of 174 (65.5\%) procedures and accurately predicted axillary nodal status in 109 of 114 (95.6\%) cases (8).

Methylthioninium chloride or Methylene Blue is an organic chloride salt. This commonly used dye is an oxidation-reduction agent and is approved by Food and Drug Administration (FDA) for the treatment of methemoglobinemia. In past, methylene blue was widely used in treatment of malaria, or as an antiseptic drug. Currently its properties are being investigated in management of patients with Alzheimer disease. It can cause acute haemolysis in patients with G6PD deficiency. When administered in the lymphatic bed of the tumor, methylene blue stains sentinel lymph nodes and is used for mapping purpose. The molecule is smaller in size than isosulfan blue. However, the specificity remains the same as for other blue dyes used for SLN mapping.

Methylene blue is less expensive than isosulfan blue or patent blue and more accessible in developing countries. The first reports about methylene blue as an alternative to isosulfan blue for sentinel lymph node biopsy in breast cancer were published by Simmons (9). The detection rate of methylene blue with radiotracer is equivalent to that reported for isosulfan and radiocolloid.

Patent blue $\mathrm{V}$ is structurally similar to isosulfan blue. The mechanism of action and biology are analogous. Both tracers are indistinctly used for Sentinel Lymph Node mapping. In some countries, like United Kingdome patent blue $\mathrm{V}$ is the most recommended blue dye in breast SLN mapping.

Indigocarmine is a blue dye used in pilot studies in Japan with promising results (10). However, more detailed data are not available on a security profile and accuracy of this method.

\section{Adverse effects of blue dyes}

Isosulfan and patent blue have been associated with anaphylactic reactions such as allergic urticaria or anaphylaxis. In the ASCOSOG Z00010 trial showed $0.1 \%$ risk of anaphylaxis using isosulfan blue alone or in combination with radiocolloid (11). Other series showed up to $1 \%$ of anaphylactic reaction. In addition, the administration of a blue dye may result in oxygen saturation decrease. This event is so called pseudo desaturation with oxygen levels remaining normal. Consequently, anaesthetic team should always be informed prior to blue dye administration.

Methylene blue has been less associated with anaphylactic reaction with only a few cases reported in the literature $(12,13)$. It can also interfere with oxygen saturation (pseudodesaturation) but less severely that isosulfan blue (14). Methylene blue causes, however, skin reactions and ulcerations. It should not be used subcutaneously due to possible skin necrosis.

Intravenous injection of blue dyes may cause causes a bluish skin discoloration. In addition, blue dyes can transitionally change the urine colour into green.

Blue dyes are contraindicated in pregnant women due to anaphylactic reaction risk. 


\section{Current indications of blue dyes}

Blue dyes are generally injected immediately before the surgery. The coloured lymph nodes are visible 5 minutes after the injection and stay up to 45 minutes. However, surprisingly some authors described injections 90 minutes prior to the surgery with positive results (15). Massage of the injection site has been described to improve the detection rate in breast cancer SLN mapping (16).

In breast cancer there is enough evidence that the combined use of blue dye and radioisotopes is significantly better than blue dye alone for SLN identification (17-19). In gynaecological cancer (endometrial, cervical and vulvar cancer) the evidence is similar. Blue dyes used alone are associated with the poorest detection rates, around $70 \%$ for endometrial cancer (50\% for bilateral detection). In vulvar cancer the sensitivity of the blue dye technique in detecting the sentinel node is only $56-88 \%$ and the combined method with radiotracer significantly improves the SLN detection rate to approximately $100 \%$.

Therefore, currently the use of blue dye alone is not recommended for SLN mapping.

\section{Indocyanine green}

Indocyanine green (ICG) is a cyanine fluorescent molecule used for diagnostic purpose in ophthalmology, neurosurgery, cardiology and hepatology. ICG has attractive characteristics due to its safety and high absorbance in a wavelength range of 600 to $900 \mathrm{~nm}$. When injected intravenously or interstitially, and excited by a laser ICG emits a wavelength in the near infrared range between 750 and $950 \mathrm{~nm}$, that can be detected by a near-infrared imaging devices. ICG is currently widely used as a sentinel lymph node tracer as after interstitial injection the drug diffuses into lymphatic pathways and lymph nodes similarly to vital dyes.

The ICG in lymph node mapping has first been used for skin cancer patients (20). Simultaneously, intraoperative near-infrared fluorescence was tested in breast cancer surgery (21-23). These first clinical trials showed similar detection rate to conventional combined technique. In 2010 ICG was first described as a feasible tracer for use in SLN mapping in gynaecologic cancers, initially cervical cancer, and then for endometrial cancer by the group of Rossi (24,25).

Recent prospective study by Frumovitz et al. proved that ICG is superior to the blue dye in detecting SLN in endometrial cancer. The authors concluded that interstitial injection of ICG appears to be safe as there were no adverse events related to the compound (26). ICG was also compared to the combined method (blue dye and radiotracer) in an international European retrospective study. The authors found significantly higher bilateral detection rate in ICG group (27). Similar studies have been performed for cervical cancer, founding better detection rate for ICG when compared to standard technique using radioisotope technetium $99 \mathrm{~m}$ radiocolloid radiotracer with or without blue dye (28). ICG is found to be the most accurate detection method for obese patients in endometrial cancer SLN mapping $(29,30)$.

The main advantage of this method is that it is a onestep technique and is fully performed during the surgical procedure what considerably impacts patient's satisfaction. ICG is associated with minimal risk of adverse effects. Although, its use is contraindicated in patients with hepatic insufficiency and those allergic to iodine components.

\section{Clinical use of ICG}

ICG may be prepared with distilled water or saline solution. However, the dosage and injection site are not standardised. Some studies used the dilution of $1.25 \mathrm{mg} / \mathrm{mL}$, using a total dosage of $5 \mathrm{mg}$, others used up to $10 \mathrm{mg}$.

An interesting experimental study used a porcine model to compare various dosages of ICG to determine the optimal dosage needed to accurately differentiate the sentinel node from surrounding tissue. The tracers have been injected into porcine uterine cervix and detected with robotic instrumentation. The authors suggest that an optimal ICG dose range of 250 to $500 \mu \mathrm{g} / \mathrm{mL}$, both of which cause a large difference in the intensity signal between the sentinel node and the surrounding tissues.

The authors found that smaller dose of ICG migrated fastest to the SLN. However, these finding have not been confirmed in different studies and the authors suggested that timing seems to be unrelated to the dosage of ICG (31).

\section{Radiotracers}

Since the development of scintigraphy and gamma camera in 1950s, radioactivity has found another application in medicine. The most widely used radiation sources for medical purposes are gamma $(\gamma)$ emitting radioisotopes, as the most penetrating and possible to be measured outside the patient's body. Only radioisotopes characterised by 


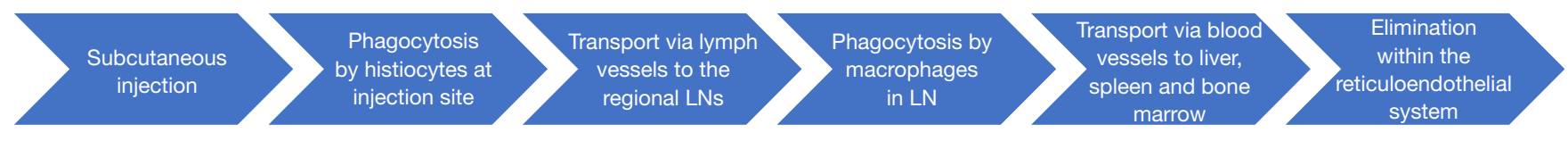

Figure 1 xxxxxxxxx

radiochemical sterility and stability, safety of application for patient and medical personnel, finally physical availability in production and costs of production not constituting a financial barrier to clinical use are classified as radiopharmaceuticals. Of the hundreds of radioisotopes available today, properties of technetium $\left({ }^{99} \mathrm{Tc}\right)$ and particularly its metastable isotope $\left({ }^{99 \mathrm{~m}} \mathrm{Tc}\right)$ gave rise to its most widely application in nuclear medicine. It emits highenergy $\gamma$ radiation and has ideal for clinical applications relatively short half-life (6.01 hours) resulting in relatively low radiation burden to the patient. It allows to precisely indicate the place of its uptake and distinguish it from the surrounding tissues. Use of molybdenum-technetium generators allows for its constant production and regular weekly delivery to hospitals as well as easy quality control process (32).

For lymph node imaging, $99 \mathrm{mTc}$ is bound to a colloid playing a role of a carrier, to form a radiocolloid. The schematic flow of the tracer in the body is shown in the Figure 1 (33).

Physical and chemical properties of colloid particle influence its kinetics and play an important role in distribution of a radiocolloid in the lymphatic vessels and ability to visualize SLN and subsequent lymph nodes. Ideal size of the colloidal particle to be used in the SLN procedure ranges between 80 and $200 \mathrm{~nm}$. Smaller elements move much more dynamic from the place of their application to the SLN, which creates a narrow window of time to determine its location, or even after passing it, subsequent nodes can be labeled leading to false results. In contrast, large particles may remain at the injection site, block lymphatic channels, or not reach the nodes within a clinically acceptable time (34). It is estimated that after 60 minutes after subcutaneous administration of the ${ }^{99 \mathrm{~m}} \mathrm{Tc}$ albumin, around $3 \%$ to $5 \%$ of the applied activity will be detected in the LNs (35). The strength of the signal will decline steadily over time as ${ }^{99 \mathrm{~m}} \mathrm{Tc}$ biological half-life time ranges between 6 and 12 hours.

There are currently over ten colloidal products used in lymphoscintigraphy following ${ }^{99 \mathrm{~m}} \mathrm{Tc}$ labeling.

Characteristics of different colloids labeled with ${ }^{99 \mathrm{~m}} \mathrm{Tc}$ and currently used in lymphoscintigraphy for the SLN procedure are presented in Table 1.

\section{Radiotracers, current indications}

Different protocols are used for different tumor sites. In cervical cancer, the most frequently used activity is about $110 \mathrm{MBq}$ in a total volume of $2 \mathrm{~mL}(0.5 \mathrm{~mL}$ per depot) (36). However, there are at least three protocols with different doses and different timing of injection. Long protocol consists of injection of high doses of radiotracer 24 hours before surgery. The doses range between 2 and $4 \mathrm{mCi}, 74-148 \mathrm{MBq}$. In short protocols, 0.2 and $1 \mathrm{mCi}$ (7.4-37 MBq) of Technetium isotope is injected 2 to 4 hours prior surgery. In ultrashort protocols the application of the is made in the operating theatre under general anaesthesia and the dose is $0.4-0.55 \mathrm{mCi}(14.8-20 \mathrm{MBq})(37)$.

The endometrial approach is more complex and several techniques have been described. Thus, the total dose injected may vary from 40 to $185 \mathrm{MBq}$ and the volume injected from 0.5 to $8 \mathrm{~mL}(38,39)$.

For vulvar cancer two to four injections of radiotracer are performed with a total dose ranging from 20 to $150 \mathrm{MBq}$ in an approximate volume of $0.4-0.5 \mathrm{~mL}(0.1 \mathrm{~mL}$ per injection) (40-42). The maximum activity of $99 \mathrm{mTc}$ should be loaded onto the smallest number of particles. Labelling at higher specific activity has been demonstrated to result in higher nodal count rates for the same administered activity (43).

Following application of a radiocolloid, lymph nodes can be localized either by planar scintigraphy or SPECT-CT. Imaging is advised to enable the preoperative identification, location and number of sentinel lymph nodes, including those outside the defined regional lymph flow. In long protocols, preoperative imaging is performed 1-3 hours after injection, whereas in short protocols the images are obtained 20-30 minutes after the injection. SPECT gamma camera and integrated CT scanner (SPECT/CT) appears to offer a significant improvement in sentinel node detection and anatomic localization when compared with lymphoscintigraphy. SPECT/CT fuses tomographic 
Table 1 Characteristics of selected ${ }^{99 \mathrm{~m}} \mathrm{Tc}-$ labelled radiopharmaceuticals used for the SLN procedure (36)

\begin{tabular}{lll}
\hline Radiopharmaceutical & Particle size (range) & Remarks \\
\hline${ }^{99 \mathrm{~m}}$ Tc albumin nanocolloid & $16-100 \mathrm{~nm}$ & Mostly used in Europe \\
${ }^{99 \mathrm{~m}}$ Tc sulfur colloid & $100-5,000 \mathrm{~nm}$ & Not filtered, not in use \\
${ }^{\text {Filtered }}{ }^{99 \mathrm{~m}}$ Tc sulfur colloid & $15-100 \mathrm{~nm}$ & Filter $0.1-0.22 \mu \mathrm{m}, \mathrm{mostly}$ used in the USA \\
${ }^{99 \mathrm{~m}}$ Tc antimony sulfide colloid & $3-30 \mathrm{~nm}$ & Mostly used in Australia, Canada \\
${ }^{99 \mathrm{~m}}$ Tc rhenium sulfide colloid & $5.1-2,200 \mathrm{~nm}$ & Used in Europe \\
${ }^{99 \mathrm{~m}}$ Tc phytate colloid & $<15-1,500 \mathrm{~nm}$ & Used in South America, Korea \\
${ }^{99 \mathrm{~m}}$ Tc tin colloid & $30-250 \mathrm{~nm}$ & Used in Korea, Japan \\
${ }^{99 \mathrm{~m}}$ Tc dextran & $10-400 \mathrm{~nm}$ & Non-colloidal, used in China and Brasil \\
\hline
\end{tabular}

lymphoscintigrams with anatomical data. SPECT/CT systems consist of a dual-head variable-angle gamma camera equipped with low-energy high-resolution collimators and a low-intensity CT scanner. Patient stays in the same position during imaging and the two images are easily fused. SPECT/CT provides a three-dimensional image with better contrast and spatial resolution than planar imaging and has the possibility to correct for attenuation and scatter. This combination of imaging properties results in precise localization of the SLN $(44,45)$.

SPECT CT has found to be particularly useful in detection of parametrial SLN in Cervical cancer $(46,47)$. However, there is still a lack of data on the use of SPECT/ $\mathrm{CT}$ in endometrial cancer. For other tumors such as vulvar cancer SPECT/CT is not widely used because deep drainage is less frequent.

Intraoperative detection of nodes with high gamma activity (hot nodes) requires a use of a gamma-probe that acquires the radioactive signal. The hot nodes are identified in comparison with background radioactivity. The ratio of radioactive counts depends on the dose, time elapsed and the type radiotracer used and type of gamma-probe applied. All hot nodes must be removed. Additionally, nodes that presents more than $20 \%$ of SLN count rate should be resected.

The quality of gamma-probe is essential for a successful mapping. The following criteria were used to describe the quality of a probe: Spatial selectivity at $30 \mathrm{~cm}$ (the width of the measurement cone), spatial resolution at $1 \mathrm{~cm}$ distance (determines the minimal distance needed between two lymph nodes for them to be detected separately, Sensitivity, Sensitivity at probe housing, energy discrimination, shape of probe (48).

\section{Safety of radiotracers}

The use of a radioactive tracer involves exposure to ionizing radiation not only for the patient but also for the medical personnel. However, operating room staff is exposed to a radiation dose reported as minimal (below $1 \mu \mathrm{Sv}$ per operation) with the highest occupational dose to the surgeons performing SLN biopsy and this is below $2 \mu \mathrm{Sv}$ per operation $(49,50)$. The hand dose to the surgeons has been calculated as 5-94 $\mu \mathrm{Sv}$ per operation. Thus, radiological safety must be secured by adherence to strict guidelines and safety rules at all stages of handling, applying and disposal of radioactive tracers

As there is limited data regarding safety of radiotracer based SLN mapping in pregnant women it is often listed as contraindication for the procedure (51). Others argue that taking into account fact that fetus exposure to radioactivity is negligible, SLN procedure is justified as is relatively related to less risks of complications than traditional axillary lymph node dissection (52).

\section{Combined methods}

The combinations of the radiotracer, ICG and blue dyes was tasted for several tumor sites in randomised trials.

In breast cancer dual detection of SLN using ICG and radioisotope was found reliable and sensitive but was not superior to isotope alone in successfully locating SLN in pilot randomized trial. Randomized comparison between indocyanine green fluorescence plus 99mtechnetium and 99 mtechnetium alone methods for sentinel lymph node biopsy in breast cancer (53).

In another prospective trial, How et al. combined the 3 tracers blue ICG and Tc99 in endometrial cancer patients. 
Authors observed a $76 \%$ bilateral detection rate using this combination, similar to single-agent ICG detection rate described in different studies (54).

The comparison of available tracers and their combination have been analysed in systematic reviews and metanalyses.

\section{New tracers}

Some authors tried to use ICG-99m Tc nanocolloid as a hybrid tracer that combined the fluorescent and radioactive signatures in one compound. These traces have been preliminary tested in melanoma, cervical and breast cancer patients (55).

Novel techniques with iron oxide tracer $\left(\right.$ Sienna $+{ }^{\circledR}$ ), have been evaluated in vulvar cancer to compare with promising results (56).

Other authors tested the fluorescence tracer with receptor targeted agents - Rituximab (a chimeric human/ murine monoclonal antibody targeting the CD20 antigen on the surface of lymphocyte for SLN mapping in breast cancer (57).

\section{Comparizon of tracers in different tumor sites}

\section{Breast cancer and melanoma}

An exhaustive meta-analysis on optimal tracer for SLN mapping in breast cancer and melanoma provided data in favor of the use of radiocolloid alone or radiocolloid combined with a blue dye. Authors of this metanalysis recommend radiocolloid alone as a method of choice for experienced surgeons (58).

\section{Vulvar cancer}

The combination of radiotracer and blue dye has for long been gold standard in SLN mapping in vulvar cancer. This opinion was based on a result of a meta-analysis which demonstrated that the SLN detection rates were $94.0 \%$ (95\% CI, 90-96\%) for technetium Tc $99 \mathrm{~m}$ alone, $68.7 \%$ (95\% CI, 63-74\%) for blue dye alone, and $97.7 \%$ (95\% CI, 96-98\%) for 99m Tc plus blue dye (59).

At present florescence guided SLN mapping is gaining importance also in vulvar cancer. Its feasibility was described in two promising studies $(60,61)$.

\section{Gynecological cancer}

According to data from a recent metanalysis, ICG or the combined technique of blue dye with Tc99 seems to have the highest detection rates, generally greater than $90 \%$ overall detection and greater than $80 \%$ bilateral detection (62).

\section{Conclusions}

The SLN procedures requires an implication of four different medical specialities including surgery, pathology, nuclear medicine and radiology. The choice of the best tracer is a result of its safety, accuracy and cost-efficacy of the method. Currently ICG is found to be the most accurate in pelvic SLN mapping both for endometrial and cervical cancer. However, its use requires availability of costly nearinfrared optical system device.

The decision of a used protocol should be made according to local availabilities and in consensus with a multidisciplinary team.

\section{Acknowledgments}

Funding: none

\section{Footnote}

Provenance and Peer Review: This article was commissioned by the Guest Editors (Denis Querleu and Cherif Akladios) for the series "Sentinel Lymph Node Biopsy in Gynecologic Cancer" published in Chinese Clinical Oncology. The article has undergone external peer review.

Conflicts of Interest: Both authors have completed the ICMJE uniform disclosure form (available at http://dx.doi. org/10.21037/cco-20-252). The series "Sentinel Lymph Node Biopsy in Gynecologic Cancer" was commissioned by the editorial office without any funding or sponsorship. The authors have no other conflicts of interest to declare.

Ethical Statement: the authors are accountable for all aspects of the work in ensuring that questions related to the accuracy or integrity of any part of the work are appropriately investigated and resolved.

Open Access Statement: This is an Open Access article 
distributed in accordance with the Creative Commons Attribution-NonCommercial-NoDerivs 4.0 International License (CC BY-NC-ND 4.0), which permits the noncommercial replication and distribution of the article with the strict proviso that no changes or edits are made and the original work is properly cited (including links to both the formal publication through the relevant DOI and the license). See: https://creativecommons.org/licenses/by-nc-nd/4.0/.

\section{References}

1. Cabanas RM. An approach for the treatment of penile carcinoma. Cancer 1977;39:456-66.

2. Fee HJ, Robinson DS, Sample WF, et al. The determination of lymph shed by colloidal gold scanning in patients with malignant melanoma: a preliminary study. Surgery 1978;84:626-32.

3. Morton DL, Wen DR, Foshag LJ, et al. Intraoperative lyphatic mapping and selective cervival lymphadenectomy for early stage melanomas of the head and neck. J Clin Oncol 1993;11:1751-6.

4. Krag DN, Weaver DL, Alex JC, et al. Surgical resection and radiolocalization of the sentinel lymph node in breast cancer using a gamma probe. Surg Oncol 1993;2:335-9.

5. Albertini JJ, Lyman GH, Cox C, et al. Lymphatic mapping and sentinel node biopsy in the patient with breast cancer. JAMA 1996;276:1818-22.

6. Wong JH, Cagle LA, Morton DL. Lymphatic drainage of the skin to a sentinel lymph node in a feline model. Ann Surg 1991;214:637-41.

7. Hirsch JI, Tisnado J, Cho SR, et al. Use of isosulfan blue for identification of lymphatic vessels: experimental and clinical evaluation. AJR Am J Roentgenol 1982;139:1061-4.

8. Giuliano AE, Kirgan DM, Guenther JM, et al. Lymphatic mapping and sentinel lymphadenectomy for breast cancer. Ann Surg. 1994;220:391-8; discussion 398-401.

9. Simmons R, Thevarajah S, Brennan MB, et al. Methylene blue dye as an alternative to isosulfan blue dye for sentinel lymph node localization. Ann Surg Oncol 2003;10:242-7.

10. Imoto $S, H a s e b e ~ T$. Initial experience with sentinel node biopsy in breast cancer at the National Cancer Center Hospital East. Jpn J Clin Oncol 1999;29:11-5.

11. Giuliano AE, Ballman KV, McCall L, et al. Effect of Axillary Dissection vs No Axillary Dissection on 10-Year Overall Survival Among Women With Invasive Breast Cancer and Sentinel Node Metastasis: The ACOSOG Z0011 (Alliance) Randomized Clinical Trial. JAMA
2017;318:918-26.

12. Rzymski P, Wozniak J, Opala T, et al. Anaphylactic reaction to methylene blue dye after laparoscopic chromopertubation Int J Gynaecol Obstet 2003;81:71-2.

13. Dewachter P, Mouton-Faivre C, Trechot P, et al. Severe anaphylactic shock with methylene blue instillation. Anesth Analg 2005;101:149-50.

14. Piñero A, Illana J, Garcia-Palenciano C, et al. Effect on oximetry of dyes used for sentinel lymph node biopsy. Arch Surg 2004;139:1204-7.

15. Song-Hua Yuan, Ying Xiong, Mei Wei, et al. Sentinel lymph node detection using methylene blue in patients with early stage cervical cancer. Gynecol Oncol 2007;106:147-52.

16. Kirby RM, Basit A, Nguyen QT, et al. Three stage axillary lymphatic massage optimizes sentinel lymph node localisation using blue dye. Int Semin Surg Oncol 2007;4:30.

17. Radovanovic Z, Golubovic A, Plzak A, et al. Blue dye versus combined blue dye-radioactive tracer technique in detection of sentinel lymph node in breast cancer. Eur J Surg Oncol 2004;30:913-7.

18. Syme DB, Collins JP, Mann GB. Comparison of blue dye and isotope with blue dye alone in breast sentinel node biopsy. ANZ J Surg 2005;75:817-21.

19. Hung WK, Chan CM, Ying M, et al. Randomized clinical trial comparing blue dye with combined dye and isotope for sentinel lymph node biopsy in breast cancer. Br J Surg 2005;92:1494-7.

20. Fujiwara M, Mizukami T, Suzuki A, et al. Sentinel lymph node detection in skin cancer patients using realtime fluorescence navigation with indocyanine green: preliminary experience. J Plast Reconstr Aesthet Surg 2009;62:e373-8.

21. Troyan SL, Kianzad V, Gibbs-Strauss SL, et al. The FLARE intraoperative near-infrared fluorescence imaging system: a first-in-human clinical trial in breast cancer sentinel lymph node mapping. Ann Surg Oncol 2009;16:2943-52.

22. Hirche C, Murawa D, Mohr Z, et al. ICG fluorescenceguided sentinel node biopsy for axillary nodal staging in breast cancer. Breast Cancer Res Treat 2010;121:373-8.

23. Murawa D, Hirche C, Dresel S, et al. Sentinel lymph node biopsy in breast cancer guided by indocyanine green fluorescence. Br J Surg 2009;96:1289-94.

24. Crane LMA,Themelis G,Pleijhuis RG, et al. Intraoperative Multispectral Fluorescence Imaging for the Detection of the Sentinel Lymph Node in Cervical Cancer: A Novel 
Concept. Mol Imaging Biol 2011;13:1043-9.

25. Rossi EC, Ivanova A, Boggess JF. Robotically assisted fluorescenceguided lymph node mapping with ICG for gynecologic malignancies: a feasibility study. Gynecol Oncol 2012;124:78-82.

26. Frumovitz M, Plante M, Lee PS. Near-infrared fluorescence for detection of sentinel lymph nodes in women with cervical and uterine cancers (FILM): a randomised, phase 3, multicentre, noninferiority trial. Lancet Oncol 2018;19:1394-403.

27. Papadia A, Zapardiel I, Bussi B, et al. Sentinel lymph node mapping in patients with stage I endometrial carcinoma: a focus on bilateral mapping identification by comparing radiotracer $\mathrm{Tc} 99 \mathrm{~m}$ with blue dye versus indocyanine green fluorescent dye. J Cancer Res Clin Oncol 2017;143:475-80.

28. Di Martino G, Crivellaro C, De Ponti E, et al. Indocyanine Green versus Radiotracer with or without Blue Dye for Sentinel Lymph Node Mapping in Stage $>$ IB1 Cervical Cancer (>2 cm). J Minim Invasive Gynecol 2017;24:954-9.

29. Tanner EJ, Sinno AK, Stone RL, et al. Factors associated with successful bilateral sentinel lymph node mapping in endometrial cancer. Gynecol Oncol 2015;138:542.

30. Eriksson AG, Montovano M, Beavis A, et al. Impact of obesity on sentinel lymph node mapping in patients with newly diagnosed uterine cancer undergoing robotic surgery. Ann Surg Oncol 2016;23:2522-8.

31. Levinson KL, Mahdi H, Escobar P. Feasibility and Optimal Dosage of Indocyanine Green Fluorescence for Sentinel Lymph Node Detection Using Robotic SingleSite Instrumentation: Preclinical Study J Minim Invasive Gynecol 2013;20:691-6.

32. Khalil MM (Ed.). Basic Sciences of Nuclear Medicine. Springer, 2011: 41-51.

33. Elgazzar $\mathrm{AH}$ (Ed.). The pathophysiologic basis of nuclear medicine. 3rd ed. Berlin and Heidelberg: Springer, 2014.

34. Jinno H, Ikeda T, Matsui A, et al. Sentinel lymph node biopsy in breast cancer using technetium-99m tin colloids of different sizes. Biomed Pharmacother 2002;56 Suppl 1:213s-216s.

35. Strand SE, Persson BR. Quantitative lymphoscintigraphy I: Basic concepts for optimal uptake of radiocolloids in the parasternal lymph nodes of rabbits. J Nucl Med 1979;20:1038-46.

36. El-Ghobashy AE, Saidi SA. Sentinel lymph node sampling in gynaecological cancers: techniques and clinical applications. Eur J Surg Oncol 2009;35:675-85.
37. Zalewski K, Benke M, Mirocha B, et al. Technetium-99mbased radiopharmaceuticals in sentinel lymph node biopsy: gynecologic oncology perspective. Curr Pharm Des 2018;24:1652-75.

38. Holub Z, Jabor A, Kliment L. Comparison of two procedures for sentinel lymph node detection in patients with endometrial cancer: a pilot study. Eur J Gynaecol Oncol 2002;23:53-7.

39. Vidal-Sicart S, Doménech B, Luján B, et al. Sentinel node in gynaecological cancers. Our experience. Rev Esp Med Nucl 2009;28:221-8.

40. Johann S, Klaeser B, Krause T, et al. Comparison of outcome and recurrence-free survival after sentinel lymph node biopsy and lymphadenectomy in vulvar cancer. Gynecol Oncol 2008;110:324-8.

41. Van der Zee AG, Oonk MH, De Hullu JA, et al. Sentinel node dissection is safe in the treatment of early-stage vulvar cancer. J Clin Oncol 2008;26:884-9.

42. Radziszewski J, Kowalewska M, Jedrzejczak T, et al. The accuracy of the sentinel lymph node concept in early stage squamous cell vulvar carcinoma. Gynecol Oncol 2010;116:473-7.

43. Ballinger JR. Effect of increased $99 \mathrm{mTc} / 99 \mathrm{Tc}$ ratios on count rates in sentinel node procedures: a randomised study. Eur J Nucl Med Mol Imaging 2004;31:306.

44. Keidar Z, Israel O, Krausz Y. SPECT/CT in tumor imaging: technical aspects and clinical applications. Semin Nucl Med 2003;33:205-18.

45. Giammarile F, Bozkurt MF, Cibula D, et al. The EANM clinical and technical guidelines for lymphoscintigraphy and sentinel node localization in gynaecological cancers. Eur J Nucl Med Mol Imaging 2014;41:1463-77.

46. Martínez A, Zerdoud S, Mery E, et al. Hybrid imaging by SPECT/CT for sentinel lymph node detection in patients with cancer of the uterine cervix. Gynecol Oncol 2010;119:431-5.

47. Zhang WJ, Zheng R, Wu LY, et al. Clinical application of sentinel lymph node detection to early stage cervical cancer. Ai Zheng 2006;25:224-8.

48. Wengenmair H., Kopp J., Sciuk J. Quality Criteria of Gamma Probes: Requirements and Future Developments. In: The Sentinel Lymph Node Concept. Berlin, Heidelberg: Springer, 2005.

49. Klausen TL, Chakera AH, Friis E, et al. Radiation doses to staff involved in sentinel operations for breast cancer. Clin Physiol Funct Imaging 2005;25:196-202.

50. Sera T, Mohos G, Papos M, et al. Sentinel lymph node detection in melanoma patients. Radiation safety 
considerations. Dermatol Surg 2003;29:141-5.

51. Lyman GH, Somerfield MR, Bosserman LD, et al. Sentinel Lymph Node Biopsy for Patients With EarlyStage Breast Cancer: American Society of Clinical Oncology Clinical Practice Guideline Update. J Clin Oncol 2017;35:561-4.

52. Giammarile F, Alazraki N, Aarsvold JN, et al. The EANM and SNMMI practice guideline for lymphoscintigraphy and sentinel node localization in breast cancer. Eur J Nucl Med Mol Imaging 2013;40:1932-47.

53. Vermersch Ch, Raia-Barjat T, Chapelle C. Randomized comparison between indocyanine green fluorescence plus 99mtechnetium and 99mtechnetium alone methods for sentinel lymph node biopsy in breast cancer. Sci Rep 2019;9:6943.

54. How J, Gotlieb WH, Press JZ, et al. Comparing indocyanine green, technetium, and blue dye for sentinel lymph node mapping in endometrial cancer. Gynecol Oncol 2015;137:436-42.

55. Paredes P, Vidal-Sicart S, Campos F, et al. Role of ICG$99 \mathrm{~m}$ Tc-nanocolloid for sentinel lymph node detection in cervical cancer: a pilot study. Eur J Nucl Med Mol Imaging 2017;44:1853-61.

56. Jedryka M, Kryszpin M, Manowiec M, et al. A new technique of sentinel lymph nodes detection in vulvar cancer patients. The sarvu study. Int J Gynecol Cancer

Cite this article as: Rychlik A, Zalewski K. Tracers and corresponding detection devices: technetium colloids, blue dyes \& NIR fluorescence. Chin Clin Oncol 2021;10(2):16. doi: $10.21037 /$ cco-20-252
2019:29:A1-A197.

57. Cong BB, Sun X, Song XR, et al. Preparation study of indocyanine green-rituximab: A new receptor-targeted tracer for sentinel lymph node in breast cancer. Oncotarget 2016;7:47526-35.

58. Niebling MG, Pleijhuisae RG, Bastiaannet E, et al. A systematic review and meta-analyses of sentinel lymph node identification in breast cancer and melanoma, a plea for tracer mapping. Eur J Surg Oncol 2016;42:466-73.

59. Covens A, Vella ET, Kennedy EB, et al. Sentinel lymph node biopsy in vulvar cancer: Systematic review, metaanalysis and guideline recommendations. Gynecol Oncol 2015;137:351-61.

60. Crane LM, Themelis G, Arts HJ, et al. Intraoperative near-infrared fluorescence imaging for sentinel lymph node detection in vulvar cancer: first clinical results. Gynecol Oncol 2011;120:291-5.

61. Schaafsma BE, Verbeek FP, Peters AA, et al. Near-infrared fluorescence sentinel lymph node biopsy in vulvar cancer: a randomised comparison of lymphatic tracers. BJOG 2013;120:758-64.

62. Ruscito I, Gasparri ML, Braicu EI, et al. Sentinel Node Mapping in Cervical and Endometrial Cancer: Indocyanine Green Versus Other Conventional Dyes-A Meta-Analysis. Ann Surg Oncol 2016;23:3749-56. 